\title{
WAVELETS based wireless VOIP and its future scenario
}

\author{
Sarvjit Singh ${ }^{1, a}$ and J.S Sohal ${ }^{2}$ \\ ${ }^{1}$ ECE Department, UIET Panjab University, Chandigarh, India \\ ${ }^{2}$ LCET, Katani Kalan, Ludhiana, India
}

\begin{abstract}
Those who use VOIP, know that a good quality service cannot be ensured over the internet as the internet is not well suited to render real time services. Besides these users do not pay much for the VOIP calls as compared to the circuit switched phone calls. So they do not bother much about the quality of services. However, it is expected that the quality should be sound enough to pay for their time as well as money. In this paper, the recently published literature has been reviewed along with the introduction to wavelets.
\end{abstract}

\section{Introduction}

Internet is most important and has already established vast converged network that supports huge communication. Initially, internet was used to transmit data only. In 1995, there was an advancement, to use internet for transmit voice traffic and video in addition to data. Now a days, Voice over Internet Protocol (VOIP) is the prominent technology used in internet upon which whole world is dependent. In VOIP one can simply transport voice signal, DTMF tones, signaling traffic, and fax over the converged network of internet. Public Switched telephone network (PSTN) is based on technology of circuit switching, which needs a dedicated channels for telecommunication; whereas VOIP is based on transmission of voice signals in form of packets, routed through packet-switched networks. Major drawback of circuit switching is rigidity as dedicated circuits remain idle during silent periods. Consequently, network resources get wasted during such periods. These networks used packet switching which transmits digitized voice data packets over the networks using multiple paths. These packets are collected at the other end to regenerate the voice signals.

In VOIP networks, network resources are not reserved, but this method gives more flexibility to the network with some congestion. Therefore, VOIP networks do not ensure voice quality to the end host. Internet is best suited converged network, but not sufficient for real time VOIP traffic applications.

\section{Element of VOIP}

Codec's (Coder/ Decoder), Packetizer and playout buffer are the three important elements of VOIP. At transmitter level voice signals are converted into desired digital signals, which are compressed and encoded into their

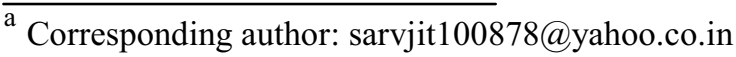

predetermined format, Figure 1 show the block diagram of VOIP system. There are many codecs available for digitizing speech, standardized by International Telecommunication Union Telecommunication (ITU-T) such as G.711, G.722, G.726, G.728, G.729, G.729(A), G.729(e), G.723.1(6.3), G.723.1(5.3), AMR [1] IS-127 codecs are defined by TIA (Telecommunications Industry Association). The packetization is carried out in which parts of encoded voice are converted into equal packet size. Furthermore, protocol headers such as Real-Time Transport protocol (RTP), User \& Datagram Protocol (UDP), Internal Protocols (IP), data link layer header from different layers are added to encoded voice packets as shown in Figure1.RTCP and RTP application layer was meant to support real time applications. TCP protocol is used in internet for less delay sensitive data packets. This introduces delay as the receiver has to notify these senders for each packet by sending acknowledgements. UDP protocol is mostly used in VOIP and other delay sensitive real time applications and it does not use scheme of acknowledgement and are faster and simpler .Therefore, more preferred for VOIP application. Then these equal size packets are transmitted over IP network to its receiver. At receiver, these received packets undergo reverse process of decoding and de-packetization. During transmission there occurs a variation in time for delivery of packets, to the receiver. This variation of time is termed as Jitter. Therefore, this Jitter can be controlled by the use of playout buffer at receiver end. Figure 1 show the block diagram of VOIP system.

VOIP also has some of signally protocols such as SIP and H.323. These signally protocols are responsible for making VOIP calls at transmitter and to end VOIP calls at Receiver [2]. H.323 signally protocol was standardized by ITU-T for smooth functionality with PSTN and used in VOIP, Internet telephony and IP based video conferencing. H.323 protocol is dependent on IETF 
(Internet Engineering Task Force), RTP and RTCP with additional protocols for call signally and data and audio visual communication. SIP is an different to H.323. It is an Application Layer signally protocol (end-to-end) to set up, modify and end multimedia sessions over the Internet. Wireless communication is the fastest growing segment. VOIP network was mainly organized by wireless technology and play a major role in next generation wireless VOIP. However, this technique is infancy before widespread use of this technology. Some urgent issues have to be settled. It is imperative to examine if wireless VOIP can provide a quality of service comparable to PSTN and cellular networks. It is also necessary to fix the number of concurrent users. A wireless network can support simultaneously without degradation of quality of service.

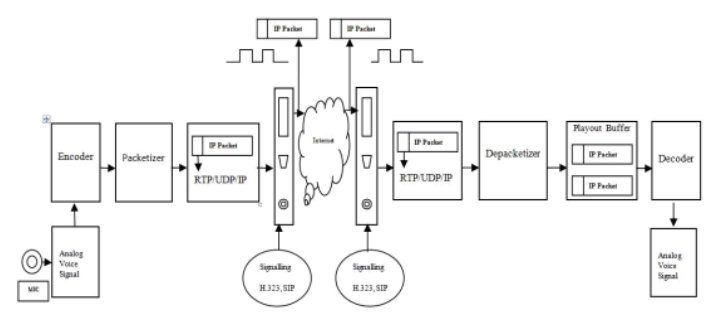

Figure 1. Block diagram of VOIP system..

To deal with this issue, first of all, we must define QOS and outline the factors effecting QOS in wireless networks. It may be referred to as a metric that measures the overall user satisfaction with the performance of the network. QOS depends on its applications and its requirements vary according to the applications. Wireless network suffers from their own merits and limitations, some of which affect performance of VOIP service. While travelling over the wireless VOIP network, voice packets must take care of factors which may affect the overall voice quality. The selection of CODEC, loss of packet, jitter and latency are the major factors that parameterized the quality of voice.

\section{Wavelets}

These could be trace back to Jean Baptiste Joseph Fourier harmonic analysis. He was the first to develop a method of describing any periodic function in terms of a weighted sum of cosines and sines. He also developed the transform that allows the expression of non -periodic time domain functions in the frequency domain, which have the scaling property and are known as Haar Wavelets family. It constitutes the simplest known wavelet set that can be used to analyze a given signal in terms of functions that are more localized in time domain than their harmonic ones. Later, Paul Levy demonstrated that Haar wavelets give better results for the study of the Brownian motion as compared to the Fourier analysis as it has a scaling property [3].

In 1982, Jean Morlet, who was the French Geophysicist gave the concept of the wavelet. A wavelet means a small wave and the study of wavelet transform is a new tool for seismic signal analysis. Then Alex Grossman studied an inverse formula for the transformation of wavelets. Jean Morlet and Alex Grossmann [4] brought out a detailed mathematical study of the continuous wavelet transform and applications their off, without realizing that similar results had earlier been available.

The effort provided a new method for breaking up a function. Wavelet may be termed as mathematical functions that sever data into different frequency components and then each component is studied with respect to a matching resolution [5].

Temporal analysis are achieved with high frequency mother wavelet, frequency analysis are carried out with a dilated low frequency wavelet. Then these set of high frequency and low frequency wavelet functions are transformed into wavelet coefficients but at different positions and different scales and final results are taken by wavelet transform of original signals [6].

Basically, wavelet analysis are made use of for the improvement of seismic signals by changing from short time Fourier analysis to an improved step by step procedure to detect sudden changes in signal reception. It does not contain any local information, the standard /classical Fourier transform are not adequate. To overcome this, Gabor transform can be used which has a feature of short-time Fourier transform by windowedFourier transform [7].

For security reasons of communication channels in VOIP and unlimited bandwidth, it necessitates to propose a need of codec and compression of audio signal to more compact form before transmission. Therefore, researchers were keen to work on this recent field to develop techniques ensuring audio coding to reduce the coded bit rate. Presently, audio compression uses wavelet transform and attracted more attention due to their encouraging $\mathrm{S} / \mathrm{N}$ ratio and compression ratio. Moreover it shows the flexibility in representing in audio signals.

\section{Literature Background}

A significant work was done on Voice over Internet Protocol and Voice over Internet Protocol using wavelets and lot of work has to be done for wireless VOIP. It was found that wavelet was an excellent tool for the analysis. It has been observed that analysis of these temporal and spectral properties of moving signals was carried out. Besides, there was developed a technique for detecting the beat attributes of music [8]. Moreover, a thresholding technique on the wavelet coefficients was also incorporated [9].A combination of wavelets and multirate filter bank in the channel coding and modulation was addressed in addition to "fractal modulation" with unpredictable communication link and in broadcast applications with QoS [10]. A new high quality, high compression approach based on AWP (Adaptive Wavelet Packet) has been proposed to achieve perceptual transform compression in [11]. Adaptive data selecting scheme for the threshold was developed for wavelet shrinkage based noise removal. Thus by considering magnitude and position of wavelet coefficient, a procedure is carried out on $2 \mathrm{D}$ cumulative sum of 
coefficient [12]. To measure the Quality for VOIP speech, a wavelet based BCF (Bark Coherence Function) was developed, which proved to be robust in VOIP applications especially to variable delay. An experimental result on VOIP speech showed that WBCF (wavelet Bark Coherence Function) performs better as compared to PSQM (Perceptual Speech Quality Measures) and BCF [13]. The loss of quality of speech over the VOIP was also examined by loss of packets and delay jitter by proposing a new formula to consider these effects in [14]. Network delay in moving time series signal was proposed which is better than AR procedure, but new DIAR (Differential Auto Regressive) algorithm was proposed which is superior to AR algorithm; it failed to extract all the stationery components from the original nonstationary series, which leads to the impression of prediction. To compensate these weaknesses, a wavelet theory was introduced into the adaptive playout procedure and proposed Wavelet Transform Auto Regressive algorithm. Finally WTAR's complexity was relatively high as compared to AR and DIAR [15].The STI (Speech Transmission Index) and SII (Speech Intelligibility Index) are the traditional intelligibility assessment methods and are not intended for VOIPPSTN channel applications. But after certain adaptations, a hypothetical solution to intelligibility assessment for NB (narrowband) speech transmission channels involving packetized networks was offered [16].A modified PCF (Point Coordination Function) i.e. DPCF (Dynamic Point Coordination Function) scheme was proposed allowing VOIP packets to be transmitted during CP only, when more than one packet is present in an STA's queue [17].The CSR (Compressed Speaker Recognition) builds selective features directly from the already compressed VOIP packet. It reduces the requirement of memory buffer and also increases the scalability [18].Then a scheme for speech hiding was proposed in which, the hiding of a secret speech into the cover speech was done to protect the secret speech from perception [19]. The performance of different audio CODECs (G.711, G.723.1, G.726 ADPCM, GSM, G.728 LC CELP, G.729 and G.729 CS-ACELP) was studied and the simulation results shows that G.723.1 audio codec in WLAN gives a least Packet end-to-end delay and Packet Delay variation and thus proved to provide an acceptable user satisfaction for WLAN VOIP [20].An adaptive steganography scheme for VOIP was proposed which proves that adaptive steganography better performs than the traditional one [21]. Since wireless means can degrade VOIP quality because of random packet losses and heavy replication be a very effective method to save these losses [22]. Then it was proved that the wavelet transform approach works better than other methods [23].VOIP capacity over WLAN and the number of calls for different voice codecs and packetization interval based on the IEEE $802.11 \mathrm{~b}$ standard was studied. At the same time, challenges for security issues were discussed and their solutions are investigated [24].A scheme based on the detection of a wavelet aimed at SIP flooding attack in VOIP network was proposed. The percentage of energy consumed according to the detailed signal was used to quickly change secret attack [25].Later on, a new steganography system for secret application was developed. It uses to hide speech files, which are impossible to differentiate [26].The delays are attributable to the difference in the jitter so we may conclude, that the data transmitted through delayed packets would not be much useful as it want add a bit to the comprehension of the message any longer. To minimize the loss the balance between size of the packet and buffer should be balanced. [27].A VOIP linking over CBR linking, over three different implementations (namely Ad-Hoc on Demand Distance Vector Routing Protocol, Fisheye Routing Protocol and Bellman-Ford Routing Protocol). The average packet lost rate and total packet sent, as seen from results considerably the packet loss portion is higher in CBR as compared to VOIP traffic generator application [28].Ethernet has much stability and insignificant delay connection as compared to wireless connection. Intervention in the neighborhood of wireless router very much reduces QOS. Consequently VOIP shows some downbeat features as it was considered a great alternative to the traditional circuit switches phones. [29].In IEEE 802.16 plan of network and MAC description which make end to end VOIP traffic video streaming possible use a network simulator. It impact is felt on QOS criterion. [30].

\section{Need of Improvement in Wireless VOIP}

Wireless communication is the fastest growing segment of communication industry. The unprecedented development of wireless system along with large number of computer based application show a very bright future for the expansion of wireless networks for VOIP. But there are many technical hurdles that challenge the designing of strong wireless network that will ensure satisfactory performance of emerging applications. There is a greater chasn between current and emerging systems which make it necessary to visualize the further wireless applications, a new reality. In this context, we must mention wireless VOIP has very promising application that may help build strong wireless network.

\section{Importance of using Wavelets in Wireless VOIP}

Over the last decade, wavelets have been a chosen field for research in wavelet based communication system. In a very short span, a much progress has been achieved in this direction. Under VOIP system audio signal is the basic unit of the signal. It is something non- stationary and of varying nature with respect to time. Such signals had temporary drifts and sudden changes. The wavelets have features of localization as well as time frequency resolution because of which these become more suitable for the transmitted audio signals through wireless VOIP applications. We have to select the most suitable wavelet adaptable to voice or data. This data is sparingly reused. This makes wavelet a very good tool for the study of wireless VOIP analysis. 


\section{Conclusions}

Future work will considered involving simulating scenarios that are closer to the real world models and work is expected to end in a wireless VOIP system with an improvement in the performance of system. This type of VOIP is viewed as a very viable substitute for PSTN. It has to satisfy the customer with high QOS. On the contrary, IP networks are still unable to fulfill the desired quality. Presently available VOIP applications which were designed for the wired environment have been improved so that wireless environment does not get degraded. With view to developing a highly improved wireless VOIP system, we will have to further investigate number of parameters so that the performance of wireless VOIP becomes matchless for the receiver.

\section{References}

1. B. Goode 'Voice over Internet Protocol (VOIP)'. Proceedings of IEEE, 90, pp. 1495-1517, (2002)

2. R. P. Swale. BT Technology Journal, 19 n.2, p.9-22, (2001)

3. C. Gargour, M. Gabrea, V. Ramachandran and J.M Lina, "A Short Introduction to Wavelets and their Applications", IEEE -CS Mag, pages 57-68, (2009)

4. A. Grossmann and, J. Morlet, SIAM Journal of Analysis, 15: 723-736, (1984)

5. M. Misiti, Y. Msiti, G.Oppenheim and J.M Poggi, "Wavelet Toolbox TM user's Guide", The math works inc; (2000)

6. R Polikar, "The Wavelet Tutorials", July16, (2001)

7. M. Sifuzzaman, M.R. Islam and M.Z. Ali, "Application of Wavelet Transform and its Advantages Compared to Fourier Transform", JPS, 13, 121-134, ISSN: 0972-8791, (2009)

8. G.Tzanetakis, G. Essl, P. Cook, "Audio Analysis using the Discrete Wavelet Transform", ICASSP, (1980)

9. Goran Kronquist and Henrik Storm, "Denoising a Speech Signal using wavelets", IEEE Transaction on Communication, 28, pp 84-95, (1990)

10. G.W. Wornell, Member IEEE, “ Emerging Applications of Multirate Signal Processing and Wavelets in Digital Communication", Proceedings of the IEEE, 84, no. 4, pages 586-603, (1996)

11. P. Srinivasan and L. H. Jamieson, "High Quality Audio Compression using an Adaptive Wavelet Packet decomposition and Psychoacoustic Modeling" IEEE Transactions on Signal Processing, 46, No. 4, pp. 1085-1093, (1998)

12. M.L. Hilton and R. T. Ogden, "Data Analysis Wavelet Threshold Selection in 2-D Signal Denoising”, IEEE Transaction on Pattern Analysis and Machine Learning, 11 pp 674-693,1999.

13. S.Wook, Y.Cheol and D.H Youn, "Speech Quality Measures for VOIP using Wavelet based Bark Coherence Function", Eurospeech Scandinavia, (2001)
14. L. Ding and R. A. Goubran, "Speech Quality Prediction in VOIP Using the Extended E-Model" 0-7803-7975-6/03/\$17.00 (C), (2003).

15. L.Zhong-bo, T. Tao, Z. S.hui, K. J.ming, "Wavelet Transform Based Adaptive Playout Algorithm for VOIP", 1zb05296@163.com.

16. F. F. Li, "Speech Intelligibility of VOIP to PSTN Interworking-A Key Index For the QoS", Printed and Published by the IEE (The institute of Electrical Engineers), pp 104-108, (2004).

17. T.Kawata, S.Shin, A.G. Forte Henning Schulzrinne, "Using Dynamic PCF to Improve the Capacity for VOIP Traffic in IEEE 802.11 Networks".

18. C.Aggarwal, D.Olshefski, D. Saha, Z.Yin Shae, P.Yu, "CSR: Speaker Recognition from Compressed VOIP Packet Stream-IBM Research Report", RC23499 (W0501-090), (2005)

19. C.Wang and Q.Wu, "Information Hiding in RealTime VOIP Streams", ninth IEEE International Symposium on Multimedia, pages 255-262, (2007)

20. I.U. Haq, K. M. Yahya, G.M.Hassan, T. M. Jadoon, "Analysis of VOIP by Using Different Vocoder in IEEE 802.11b Wireless Networks", NCSCCKKNWFP, Pakistan, May 23-25, pages 84-87, (2008)

21. H.Tian, K.Zhou, H.Jiang, Y. Huang, J. Liu, and D.Feng, "An Adaptive Steganography Scheme for Voice over IP" IEEE ISCAS 2009.

22. A. Mondal, C.Huang, J.Li, M.Jain and A. Kuzmanovic, "A Case for Wifi Relay: Improving VOIP Quality for Wifi Users".

23. M. Sifuzzaman, M.R. Islam and M.Z. Ali, "Application of Wavelet Transform and its Advantages Compared to Fourier Transform", JPS, 13, 121-134 ISSN: 0972-8791, (2009)

24. H.Kazemitabar, S.Ahmed, K.Nisar, A.B. Said and H.B. Hasbullah, "A Comprehensive review on VOIP over Wireless LAN networks", www.csl.issres.net, 2(2), (2010)

25. J. Tang, Y Cheng, "Quick Detection of Stealthy SIP Flooding Attacks in VOIP Networks", Proceedings of IEEE ICC, Kyoto, Japan, (2011).

26. S.Rekik, D.Guerchi, S.A.Selouani and H.Hamam, "Speech steganography using wavelet and Fourier Transforms", EURASIP Journal on Audio, Speech, and Music Processing 2012, A Springer Open Journal, (2012)

27. I.Philipa, V.E Idigo, A.James, "Modeling and Simulation of Voice over Internet Protocol (VOIP)", (IJEIT) 1, Issue 4, ISSN: 2277-3754, pp 225-233, (2012)

28. R.Ganiga, B.Muniyal and Pradeep, "International Journal of Computer Applications (0975 - 8887)" 50 - No.11, pp 25-31, (2012).

29. Y.Pan, J.Chung, Z. Zhang, “Analysis of Performance of VOIP Over various scenarios OPNET 14.0”, Final Report Group 11, SFU, Spring (2012).

30. A. Dubey, "Quality of Service (QoS) in Wireless Network and NS-3, VOIP Simulation Environment", (IJSCE) ISSN: 2231-2307, 2, Issue-5, (2012). 